\title{
Tratamiento adyuvante y de rescate tras prostatectomía radical
}

\author{
J.L. Mengual Cloquell, P.P. Escolar Pérez, M. Casaña Giner, M.L. Chust Vicente, \\ J.L. Guinot Rodríguez, L. Arribas Alpuente
}

Servicio de Oncología Radioterápica. Fundación Instituto Valenciano de Oncología. Valencia.

Actas Urol Esp 2005; 29 (6): 553-561

\section{RESUMEN}

TRATAMIENTO ADYUVANTE Y DE RESCATE TRAS PROSTATECTOMIA RADICAL

Tras la prostatectomía radical es importante identificar pacientes con alto riesgo de enfermedad microscópica residual en ausencia de enfermedad micrometastásica. La radioterapia adyuvante en estudios retrospectivos reduce las recurrencias locales y es más eficaz que la RT de rescate, aportando mejor supervivencia libre de recaída bioquímica y un potencial impacto en la supervivencia global. La supresión androgénica puede posiblemente eliminar la enfermedad oculta y aportar un efecto aditivo sobre el control local.

Es posible identificar y seleccionar los pacientes que pueden beneficiarse de la Radioterapia de rescate, incluso los de alto riesgo. Se puede determinar la predicción de la progresión de PSA tras rescate con radioterapia y el riesgo de metástasis.

Analizamos la bibliografía para un mejor enfoque terapéutico, basándonos en los datos actuales conocidos, a la espera de realización de nuevos ensayos clínicos.

Palabras clave: Prostatectomía radical. Radioterapia adyuvante y de rescate. Hormonoterapia.

\section{ABSTRACT}

\section{ADYUVANT AND SALVAGE RADIOTHERAPY AFTER RADICAL PROSTATECTOMY}

After radical prostatectomy is important to identify patients who have a high risk of microscopic residual disease without micrometastatic disease. Adyuvant RT, in retrospective studies, reduce the risk of recurrence and is more efficacious than salvage RT and can improve PSA relapse-free survival and should have an impact on long-term overall survival. The benefit of androgen suppresion could be due to a synergistic interaction and may possibly eliminate occult systemic disease.

Appropiate selection to identify subgroups of patients who may benefit from salvage RT, even for those patients at the highest risk; and whether some form of hormone ablation should accompany. To predict the biochemical failure and the risk of metastasic disease after salvage RT.

We analize the references to selection an appropiate therapy. Improves outcomes will need to be tested in randomized trials.

Keywords: Radical Prostatectomy. Adyuvant and salvage RT. Hormonal therapy.

$\mathrm{L}^{2}$ a prostatectomía radical con linfadenectomía es una de las posibles alternativas de tratamiento de los tumores de próstata órgano-confinados (cT1/cT2 N0 M0), junto con la braquiterapia (implantes de semillas permanentes) y la radioterapia externa con escalada de dosis (3DIMRT).
$\mathrm{Al}$ analizar la pieza quirúrgica se produce un supraestadiaje hasta en un 50\% de los pacientes. Los factores histológicos desfavorables podrán determinar los pacientes candidatos a tratamiento adyuvante con RT exclusiva o asociada a hormonoterapia. La incidencia de afectación extracapsular oscila entre el 30-65\%, la de vesículas 
seminales entre un $10-15 \%$ y la presencia de márgenes quirúrgicos positivos entre el $5-50 \%{ }^{1,2}$. Los pacientes con alta probabilidad de enfermedad residual microscópica tras la prostatectomía tienen un $50 \%$ de riesgo de fallo bioquímico a los 5 años y la mitad de estos pacientes desarrollarán metástasis a los 7-8 años ${ }^{3}$.

La radioterapia adyuvante en estudios retrospectivos reduce las recurrencias locales ${ }^{4}$ y según Taylor y col. es más eficaz que la de rescate, aportando mejor supervivencia libre de recaída bioquímica ${ }^{5}$.

El nivel de PSA tras cirugía tiene que ser indetectable $(<0,1 \mathrm{ng} / \mathrm{ml})$ en el plazo de semanas, la persistencia implica presencia de enfermedad residual.

La progresión bioquímica es definida como PSA $>0,1-0,2 \mathrm{ng} / \mathrm{ml}$ tras un período de PSA indetectable. Entre el 27-53 \% de los pacientes sometidos a prostatectomía presentarán elevaciones de PSA dentro de los 10 primeros años tras la cirugia $^{1,3}$, hasta el $23 \%$ de los que recaen presentarán niveles de PSA indetectables durante 5 años y el $4 \%$ comenzará a elevarse el PSA trascurridos 10 años ${ }^{3}$.

La evolución tras la recaída bioquímica puede ser hacia una recaída local o hacia enfermedad diseminada.

La mediana de tiempo actuarial, desde el fallo bioquímico sin tratamiento hasta la aparición de metástasis, es de 7,5-8 años ${ }^{3,6}$ y de 5-6,5 años entre la aparición de metástasis y la muerte ${ }^{3,6}$.

La introducción del tratamiento con radioterapia y hormonoterapia sigue siendo tema de controversia, pero existen numerosos estudios que sugieren mejores resultados con radioterapia de rescate si se aplica de forma precoz.

Es importante analizar factores de riesgo para predicción de fallos locales y de progresión sistémica. Los pacientes con alto grado de enfermedad, corto intervalo de supervivencia libre de enfermedad desde la prostatectomía y rápido incremento de PSA (tiempo duplicación 6-10 meses), presentan un alto riesgo de progresión a distancia ${ }^{3}$. La radioterapia de rescate es efectiva para conseguir respuestas de PSA y mejorar la supervivencia libre de progresión ${ }^{7}$. No hay estudios previos que demuestren que la RT de rescate mejore la supervivencia global o prevenga la enfermedad a distancia, pero un nuevo estudio de Stephenson et al. ${ }^{8}$, sugiere que puede modificar la historia natural de los pacientes con recurrencia de PSA incluso en los pacientes con alto riesgo de enfermedad a distancia.

Se requieren estudios randomizados para investigar si la radioterapia de rescate puede prevenir las metástasis a distancia e incrementar la supervivencia en los pacientes con recaídas tras la prostatectomía.

Las posibles situaciones clínicas que nos podemos encontrar en los trabajos o ensayos clínicos de RT adyuvante es la de PSA indetectable con factores pronósticos desfavorables y en los de RT de rescate:

- PSA persistente (no $<0,1 \mathrm{ng} / \mathrm{ml}$ ) tras cirugía.

- PSA>0,2 ng/ml con PSA previo indetectable

= Recaída bioquímica.

- Recaída Local con biopsia positiva.

\section{TRATAMIENTO ADYUVANTE CON FACTORES PATOLOGICOS DE ALTO RIESGO}

PSA INDETECTABLE con factores pronósticos desfavorables:

La primera pregunta que nos plantearemos: ¿Qué actitud terapéutica hay que adoptar tras la prostatectomía?

- No tratamiento adyuvante. Seguimiento.

- Radioterapia adyuvante exclusiva.

- Radioterapia + hormonoterapia adyuvante.

- Hormonoterapia adyuvante.

Es necesario identificar pacientes con alto riesgo de enfermedad microscópica residual en ausencia de enfermedad micrometastásica.

La radioterapia adyuvante sobre la celda prostática, en estas situaciones de alto riesgo de persistencia de enfermedad subclínica y por tanto de fallo bioquímico o clínico ${ }^{9,10}$ : PSA inicial > $10 \mathrm{ng} / \mathrm{ml}$, Gleason 5 o mayor, márgenes positivos, afectación extracapsular y de vesículas seminales sigue como tema de debate. El riesgo de fallo bioquímico a los 5 años es del 40$60 \%$ cuando existe positividad de márgenes quirúrgicos ${ }^{9-12}$, considerándose como un factor predictor de enfermedad recurrente tras la prostatectomía.

D'Amico et al. ${ }^{13}$, en 1095 pacientes tratados con prostatectomía radical, el estadio clínico ini- 
cial de T2, PSA inicial al diagnóstico $>10 \mathrm{ng} / \mathrm{ml}$, velocidad anual de PSA preoperatorio $>2 \mathrm{ng} / \mathrm{ml}$, Gleason 8-10 de la biopsia, todos estos factores fueron asociados con un intervalo más corto de aparición de recurrencia y a un incremento del riesgo relativo de mortalidad por cáncer de próstata o por otra causa.

Varios estudios ${ }^{4,14-18}$ han demostrado que la radioterapia adyuvante aporta un beneficio en la reducción del riesgo de fallo de PSA entre 30-88\% a los 5 años, un incremento del control local y de la supervivencia libre de enfermedad en seleccionados pacientes de alto riesgo pero no queda claramente definido el impacto en la supervivencia global $^{4}$. La mitad de los pacientes con invasión de las vesículas seminales (pT3b) que son tratados con RT adyuvante permanecen libres de enfermedad a los 5 años ${ }^{5,19}$

Anscher et al. ${ }^{4}$, analizan una serie de 159 pacientes $\mathrm{pT} 3 / \mathrm{T} 4$ tras prostatectomía radical:

- 113 pacientes no recibieron RT adyuvante, con una mediana de seguimiento de 10 años objetivan una supervivencia libre de enfermedad del $37 \%$ a los 10 años y del 33\% a los 15 años.

- Los 46 pacientes que recibieron RT adyuvante, con un rango de dosis entre 55-66 Gy, presentaron una supervivencia libre de enfermedad del 55\% a los 10 años y del $48 \%$ a los 15 años. La incidencia de fallos locales fue del $40 \%$ en el grupo de cirugía exclusiva frente al $8 \%$ en el grupo de RT adyuvante.

Valicenti et al. ${ }^{14}$, en un grupo de 149 pacientes pT3 y con PSA indetectable: 97 pacientes fueron solo tratados con cirugía hasta la recaída de PSA, objetivando una supervivencia libre de recaída bioquímica del 55\% a los 5 años. Los 52 pacientes tratados con RT adyuvante dentro de los 3-6 meses tras cirugía consiguieron una supervivencia libre de recaída bioquímica del $89 \%$ a los 5 años, objetivando una reducción del $88 \%$ del riesgo de recaída de PSA.

Taylor et al. ${ }^{5}$ en un grupo de 75 pacientes con PSA post-cirugía $<0,1 \mathrm{ng} / \mathrm{ml}, 27$ pT1-2 y 48 pT34 , con factores de alto riesgo: márgenes positivos en $73 / 75$, vesículas seminales afectas en $13 / 75$ y con afectación extracapsular en $45 / 75$, fueron tratados con RT adyuvante con una mediana dosis de 60 Gy , en 2 se asoció ablación androgé- nica .La mediana de tiempo desde la prostatectomía hasta el inicio de la RT fue de 5 meses (rango 1-12 meses). De los 75 pacientes, 8 desarrollaron progresión de PSA (10,6\%), con una supervivencia libre de recaída bioquímica (SLRB) del 88\% a los 5 años. Analizando según los factores de riesgo, la SLRB en los que tenían infiltración de vesículas seminales baja al 65\% comparada con el 94\% cuando no se encontraba afecta. Los que tenían Gleason 8-10 fue del 77\% frente al 96\% en los de Gleason 2-7. Si el PSA inicial fue $>10$ $\mathrm{ng} / \mathrm{ml}$ obtuvieron el $76 \%$ frente al $100 \%$ en los de $\mathrm{PSA}</=10$.

Un estudio Fase III (22911) de la EORTC (cerrado en diciembre 2001), pendiente de resultados definitivos, incluía pacientes pT3 pNO con hallazgos patológicos de invasión capsular, márgenes quirúrgicos positivos y afectación de vesículas seminales; randomiza no más tratamiento tras cirugía frente a irradiación pélvica postoperatoria de $60 \mathrm{~Gy}$. El objetivo principal era determinar la supervivencia libre de progresión bioquímica y secundariamente analizar control local y loco-regional, supervivencia libre de progresión clínica, supervivencia global y causa específica y finalmente toxicidad aguda y tardía. En los resultados preliminares ${ }^{20}$, la adición de RT adyuvante post-operatoria aporta un incremento de la supervivencia clínica o biológica a los 5 años con respecto a los pacientes tratados con cirugía y seguimiento e irradiación pélvica en caso de recaída: $72,2 \%$ vs $51,8 \%$ con una toxicidad tardía Grado 3 del 2,8\%.

El ensayo Fase III randomizado de la RTOG P0011 de tratamiento adyuvante para pacientes con alto riesgo pT2-3 NO MO y PSA previo a cirugía $</=40$, incluye:

- pT2 con Gleason $>/=7$, con PSA precirugía $>$ 10 y márgenes + .

- pT3 con Gleason >/=7 y uno ó más de los siguientes factores: PSA precirugía $>10$, márgenes positivos, vesículas seminales afectas. - pT3 con Gleason < 7 y 2 o más factores de los anteriormente mencionados.

El estudio randomiza 3 brazos: a) análogos LH-RH durante 2 años y 1 mes de antiandrógeno más RT con una dosis entre 63-66 Gy. b) RT exclusiva. c) agonistas LH-RH durante 2 años (este brazo cerrado en diciembre 03). 
El objetivo principal es ver si la adición de la supresión androgénica en los pacientes de alto riesgo mejora la supervivencia global de la RT exclusiva. Otros objetivos son determinar la supervivencia libre de enfermedad, supervivencia libre de PSA y supervivencia libre de metástasis.

El estudio Fase III randomizado prospectivo del M.D. Anderson Cancer Center (ID 97-077) intenta dilucidar la aportación de la Hormonoterapia adyuvante tras la prostatectomía con PSA < $0,1 \mathrm{ng} / \mathrm{ml}$ e incluye pacientes con alto riesgo de recaída bioquímica y que presente al menos uno de los siguientes criterios:

- Gleason $>/=8$,

- invasión de vesículas seminales .T3b

- extensión extraprostática con márgenes positivos y (Gleason $>/=7$ ).

Quedamos a la espera de resultados de los estudios actuales randomizados Fase III y de una nueva propuesta de estudio randomizado $(2204 x)$ de la EORTC para determinar si la adición de Hormonoterapia a la radioterapia adyuvante post-operatoria de 66 Gy mejora la supervivencia libre de PSA, la de progresión clínica y la global. La supresión androgénica puede posiblemente eliminar la enfermedad oculta y aportar un efecto aditivo sobre el control local, sabiendo que ya es efectiva para la enfermedad macroscópica en los estadios avanzados.

\section{TRATAMIENTO DE RESCATE}

Los trabajos o ensayos de RT de rescate incluyen pacientes con:

- PSA persistente (no $<0,1 \mathrm{ng} / \mathrm{ml}$ ) tras cirugía.

- Recaída bioquímica $=$ PSA $>0,2 \mathrm{ng} / \mathrm{ml}$ con PSA previo indetectable:

- Recaída local con biopsia positiva.

La RT de rescate potencialmente puede curar a los pacientes con enfermedad recurrente tras la prostatectomía, pero estudios previos parecen evidenciar que es inefectiva en el grupo de pacientes con alto riesgo de enfermedad diseminada. La RT rescate obtiene entre el 30-70\% de supervivencia libre de recaída bioquímica a los 3-5 años ${ }^{17,21}$.

Taylor et al. ${ }^{5}$ en un grupo de 71 pacientes tratados con RT rescate y una mediana dosis de 70 Gy:

- incluye 27 pacientes con persistencia PSA tras prostatectomía, con una mediana de PSA de 0,4 ng/ml (rango 0,1-18), en 12/27 además se asoció ablación androgénica.
- 44 pacientes con recaída de PSA y una mediana de PSA de $0,8 \mathrm{ng} / \mathrm{ml}$ (rango $0,1-3$ ), en 14/44 presentaban recurrencia local confirmada con biopsia y en 23/44 se asoció con ablación androgénica.

La supervivencia libre de recaída bioquímica a los 5 años es del 66\%. Se presentaron 20/71 fallos bioquímicos. En el grupo de pacientes de persistencia de PSA obtiene una supervivencia libre de PSA del 43\% frente al 78\% del grupo de recaída PSA. Los 35 pacientes con ablación hormonal y con una mediana de duración de tratamiento de 2 años presentaron una supervivencia libre de PSA a los 5 años del 81\% frente al 54\% de los 36 restantes no sometidos a ablación androgénica.

Stephenson et al. ${ }^{8}$, analizan una serie de 501 pacientes, tratados con $\mathrm{RT}$ de rescate sobre celda prostática con una mediana de dosis de 64,8 Gy (rango 3780-7560 cGy), con el objetivo de determinar que pacientes pueden beneficiarse de la misma e identificar variables pronósticas asociadas a una mayor duración de la respuesta: 481/501 pacientes $(96 \%)$ con $\mathrm{PSA}=$ ó $>0,2 \mathrm{ng} / \mathrm{ml}$ y 20 pacientes (4\%) recibieron tratamiento antes de alcanzar 0,2 $\mathrm{ng} / \mathrm{ml}$ pero con al menos dos incrementos consecutivos de PSA a los niveles de $0,1 \mathrm{ng} / \mathrm{ml}$ o mayor. En 160 pacientes (32\%) persistían niveles detectables de PSA tras la prostatectomia radical. En 105 pacientes (21\%) existía recidiva local confirmada por biopsia. Recibieron hormonoterapia neoadyuvante 83 pacientes (17\%), con una mediana de duración de 3 meses (rango 1-6 meses).

La mediana de seguimiento fue de 45 meses, 250 de los 501 pacientes presentaron enfermedad progresiva (50\%), 49 desarrollaron enfermedad metastásica (10\%), 20 murieron por su cáncer próstata (4\%), 21 fallecieron por enfermedad intercurrente (4\%). La supervivencia actuarial global a los 7 años es de $82 \%$ y la causa específica del 90\%. La probabilidad de estar libre de progresión, tras la RT de rescate, a los 4 años fue del $45 \%$.

Selección subgrupos según factores pronósticos Predicción buena respuesta a la radioterapia de rescate

Tenemos la posibilidad de identificar los distintos factores pre y post-quirúrgicos tanto bioquímicos, clínicos y patológicos de valor pronóstico y predictores de respuesta: 
1. PSA inicial previo a prostatectomía. Velocidad anual PSA preoperatorio.

2. Gleason 4-7/8-10.

3. T3a/T3b.

4. Márgenes negativos.

5. Cambios del PSA postoperatorio: PSA preRT, tiempo duplicación PSA, velocidad PSA, pendiente PSA.

Tras la recogida y análisis de todos estos factores podemos seleccionar los pacientes que pueden beneficiarse de la RT de rescate, incluso los de alto riesgo y a los que es adecuado asociar Hormonoterapia a la RT. Además estos factores nos facilitan la predicción de la progresión de PSA tras rescate con radioterapia y la predicción del riesgo de metástasis.

D'Amico et al. ${ }^{13}$, encuentran que los pacientes, con una velocidad de PSA preoperatoria anual $>$ de $2 \mathrm{ng} / \mathrm{ml}$, pueden tener tiempos más cortos de aparición de recurrencia, mayor riesgo relativo de mortalidad por cáncer de próstata o muerte por otra causa.

La presencia de márgenes quirúrgicos positivos se considera como un factor predictor de una buena respuesta a la $\mathrm{RT}$ de rescate $^{7}$, pone de manifiesto que la recurrencia es secundaria a enfermedad residual pélvica. Más de la mitad de los pacientes con márgenes positivos y Gleason 8-10 pueden beneficiarse de una larga respuesta a la Radioterapia de rescate.

Unas cifras elevadas del PSA pre-radioterapia tienen una influencia negativa en los resultados de la radioterapia de rescate $^{21}$, considerándose en múltiples estudios que el rango entre 0,6-4 $\mathrm{ng} / \mathrm{ml}$ es el corte para incluir en ensayos clínicos pacientes y poder predecir una mejor respuesta a la RT de rescate ${ }^{7}$. Es adecuado dividir en grupos según las cifras de PSA pre-radioterapia según sea $<$ ó $=1 \mathrm{ng} / \mathrm{ml}$, entre $1-2 \mathrm{ng} / \mathrm{ml} \mathrm{y}>2 \mathrm{ng} / \mathrm{ml}$.

La American Society for Therapeutic Radiology and Oncology (ASTRO) considera que la cifra de corte adecuada del nivel de PSA pre-RT es de 1,5 $\mathrm{ng} / \mathrm{ml}$. La probabilidad de supervivencia actuarial libre de progresión a los 4 años cuando el PSA pre-RT es $>2 \mathrm{ng} / \mathrm{ml}$ es del $12-25 \%$.

El tiempo de duplicación del PSA (TDPSA), es uno de los factores pronósticos y predictor de tiempo más importante de progresión local, sistémica y de mortalidad causa específica. La media- na de supervivencia tras fallo de PSA en pacientes con TDPSA $<3$ meses es de 6 años. Los tiempos de duplicación mayor de 6-12 meses, primariamente el riesgo será de recaída local y si es menor de 6-10 meses, mayor riesgo de enfermedad metastásica.

Roberts et al. ${ }^{22}$, en un estudio de 879 fallos bioquímicos (31\%) tras 2.809 prostatectomías, identifica que el tiempo de duplicación del PSA es el más importante factor pronóstico de recaída local y sistémica. El porcentaje de pacientes con TDPSA $<6$ meses fue más alto si la primera presentación clínica fue como progresión sistémica (48\%) comparado con recurrencia local (18\%). La correlación del TDPSA $^{22}$ con la tasa media de fallos locales, recurrencia local y enfermedad sistémica viene expresada en la Tabla 1.

Tabla 1

Correlación TDPSA con tasa fallos

\begin{tabular}{lccc}
\hline TDPSA & $\begin{array}{c}\text { Fracasos } \\
\text { globales }\end{array}$ & $\begin{array}{c}\text { Recurrencia } \\
\text { local }\end{array}$ & $\begin{array}{c}\text { Progresión } \\
\text { sistémica }\end{array}$ \\
\hline$>10$ años & $13 \%$ & $12 \%$ & $1 \%$ \\
$1-9,9$ & $38 \%$ & $33 \%$ & $5 \%$ \\
$0,5-0,9$ & $54 \%$ & $47 \%$ & $7 \%$ \\
$<0,5$ años & $62 \%$ & $26 \%$ & $36 \%$ \\
\hline
\end{tabular}

Por tanto los pacientes, con alto riesgo de recurrencia local, podrán beneficiarse de RT local y los de alto riesgo de enfermedad metastásica requerirán tratamiento sistémico asociado.

Katz et al. ${ }^{7}$, identifican factores de predicción de respuesta bioquímica tras RT rescate con 66 Gy, en un grupo de 116 pacientes con fallo bioquímico, con una mediana de intervalo desde la prostatectomía de 27 meses y una mediana de PSA pre-RT de $0,87 \mathrm{ng} / \mathrm{ml}$. Los factores de riesgo son PSA >0,6 $\mathrm{ng} / \mathrm{ml}$, márgenes negativos, Gleason $>7$ y afectación de vesículas seminales. La supervivencia actuarial libre de recaída PSA es del $0 \%$ a los 4 años cuando se asocian 3 ó 4 factores, del $21,8 \%$ con 2 , del $73 \%$ con 1 y del $94,4 \%$ si no existen factores de riesgo.

En el estudio de Stephenson et al. ${ }^{8}$, en un grupo de 356/501pacientes que no recibieron hormonoterapia neoadyuvante y recogidos todos los datos, la probabilidad de supervivencia actuarial libre de progresión (PLP) a los 4 años según subgrupos de pacientes y factores pronósticos es expresada en la Tabla 2. 
Tabla 2

Probabilidad de supervivencia actuarial libre de progresión según subgrupos de pacientes y factores pronósticos

\begin{tabular}{|c|c|c|c|c|c|c|c|}
\hline Gleason 4-7 & $\begin{array}{c}\text { Gleason } \\
8-10\end{array}$ & PSA $\triangleleft=2$ & PSA $>2$ & Margen & $\begin{array}{l}\text { TDPSA> } \\
10 \text { Meses }\end{array}$ & $\begin{array}{l}\text { TDPSA } \angle 1= \\
10 \text { Meses }\end{array}$ & PLP\% \\
\hline+ & & & & & & & 50 \\
\hline+ & & + & & & & & 58 \\
\hline+ & & + & & + & & & 69 \\
\hline+ & & + & & + & + & & 77 \\
\hline+ & & + & & + & & + & 64 \\
\hline+ & & & + & & & & 22 \\
\hline+ & & + & & - & & & 43 \\
\hline+ & & + & & - & + & & 69 \\
\hline \multirow[t]{8}{*}{+} & & + & & - & & + & 22 \\
\hline & + & & & & & & 29 \\
\hline & + & + & & & & & 35 \\
\hline & + & + & & + & & & 51 \\
\hline & + & + & & + & + & & 81 \\
\hline & + & + & & + & & + & 37 \\
\hline & + & + & & - & & & 18 \\
\hline & + & & + & & & & 12 \\
\hline
\end{tabular}

Si analizamos la tabla vemos que la probabilidad de estar libre de progresión (PLP) se va incrementando a medida que se van asociando al Gleason los factores de buen pronóstico, obteniendo hasta el $77 \%$ cuando se combinan Gleason 4-7, PSA pre-RT < ó =2, márgenes positivos y tiempo duplicación PSA > 10 meses; y hasta el $81 \%$ cuando se asocian Gleason 8-10 con idénticos factores.

-Si el Gleason 8-10 con márgenes positivos y PSA pre-RT $<2$ pero con Tiempo de duplicación $<10$ meses, la PLP es del 37\%.

- Si el Gleason 8-10 va asociado a márgenes negativos aunque el PSA pre-RT sea menor de 2, la PLP baja al 18\% y cuando se asocia a PSA preRT $>2$ disminuye al 12\%.

- Si el Tiempo de duplicación PSA es < ó $=10$ meses y va asociado a los otros 3 factores de buen pronóstico (Gleason 4-7, PSA < 2 y márgenes positivos) obtiene una PLP del 64\%.

- Cuando el Tiempo de duplicación PSA es < 10 meses y con márgenes negativos aunque se asocie con Gleason 4-7 y PSA pre-RT < 2, la PLP baja al $22 \%$.

- Si los márgenes negativos van asociados a Gleason 4-7, PSA pre-RT <2 y con Tiempo duplicación de PSA > 10 meses obtiene una supervivencia libre de progresión (PLP) del 69\%.
- El PSA pre-RT > 2 implica baja probabilidad de estar libre de progresión, asociado al Gleason 4-7 es del 22\% y del $12 \%$ si Gleason 8-10.

Por tanto los pacientes con Gleason 8-10, márgenes negativos, tiempo duplicación de PSA < 10 meses, PSA pre-RT $>2 \mathrm{ng} / \mathrm{ml}$ presentan un alto riesgo de enfermedad progresiva y escasas posibilidades de mantener una respuesta duradera con la Radioterapia de rescate de forma exclusiva ${ }^{8}$.

\section{FALLO BIOQUIMICO. POSIBILIDADES DE TRATAMIENTO}

Tras constatar el fallo bioquímico nos preguntaremos a) ¿qué pauta de tratamiento es la más adecuada? b) ¿Cuál es el momento óptimo y los valores de PSA adecuados para introducir el tratamiento adyuvante?

1. No tratamiento. Observación hasta recaída local o aparición de enfermedad metastási$\mathrm{ca}^{6}$.

2. Seleccionar/identificar subgrupos de pacientes para tratar con Radioterapia exclusiva $^{8}$.

3. Asociar radioterapia con hormonotera$\mathrm{pia}^{23,24}$.

4. Introducción precoz o no de Hormonoterapia $^{25,26}$. 


\section{No tratamiento. Observación}

Eisenberger ${ }^{6}$, en una serie de 329 pacientes con fallo bioquímico tras prostatectomías, fueron observados con seguimiento clínico y radiológico. Analiza la historia natural del fallo bioquímico no tratado, con una mediana de seguimiento de 10,5 años. El tiempo mediano actuarial hasta la aparición de metástasis es de 7,5 años y desde la diseminación hasta la muerte es de 6,5 años. También analiza los factores predictivos de supervivencia libre de metástasis según:

- tiempo de duplicación del PSA $</=10$ meses ó > 10 meses.

- si la presentación de la recurrencia se produce dentro de los 2 años tras cirugía o es más tardía.

- grado de Gleason.

Cuando el tiempo duplicación del PSA es $</=10$ meses, la supervivencia libre de metástasis a los 3 años es de 52-66\%; a los 7 años del 6-7\% para todos los Gleason si la recurrencia se produce dentro de los 2 años tras cirugía, y del $24-27 \%$ para recurrencias más tardías (> 3 años).

Si el tiempo de duplicación del PSA es $>10$ meses, la supervivencia libre de metástasis a los 3 años es del 84-99\%; a los 7 años es del 57\% para Gleason $>/=8$ y del 75-84\% para Gleason 5-7.

El grado de Gleason y tiempo de duplicación corto predice de forma significativa la aparición de enfermedad metastásica.

Selección subgrupos según factores pronósticos predicción buena respuesta a la radioterapia de rescate (desarrollado previamente).

\section{Asociar $R T$ rescate con hormonoterapia adyuvante}

Tras la selección de los subgrupos de pacientes, parece adecuada la asociación de HT adyuvante a los pacientes con baja probabilidad de estar libre de progresión con RT exclusiva y con factores pronósticos negativos. El ensayo Fase III RTOG-9601(cerrado) de Radioterapia con 64,8 Gy sobre volumen inicial de la próstata, randomiza Bicalutamida durante 2 años frente a placebo en pacientes con elevación del PSA tras prostatectomías e incluye pacientes con PSA entre 0,3-4 ng/ml, estadío patológico pT3 NO o pT2 NO con márgenes positivos o T2 sin márgenes positivos pero con biopsia de celda prostática o anastomosis $^{23}$.

King et $a .^{24}$, en un estudio retrospectivo de 122 pacientes con fallos de PSA tras prostatectomía asocian en 53 pacientes bloqueo androgénico completo durante 2 meses previos y 2 meses concurrentes con la RT de rescate, obtienen una supervivencia libre de fallo bioquímico del $57 \%$ a los 5 años y una supervivencia global del 100\% a los 5 años. En los restantes 69 pacientes que solo recibieron RT la supervivencia libre de fallo bioquímico baja al $31 \%$ a los 5 años y la supervivencia global al $87 \%$ a los 5 años. La hormonoterapia asociada a la RT posiblemente aporte mejoría en la supervivencia global.

\section{Introducción precoz hormonoterapia}

El beneficio de la hormonoterapia en los pacientes con recaída bioquímica no queda claro, así como el momento óptimo de introducirla.

W. Reynolds et al. ${ }^{25}$ en un grupo de 4227 pacientes tratados, entre 1988-2002, con prostatectomía radical, con una mediana de seguimiento de 3.9 años, 536 pacientes presentaron recurrencia de PSA durante el primer año. Los pacientes sometidos a bloqueo androgénico precoz tenían mejor supervivencia libre de metástasis comparado con los no bloqueados o bloqueados de forma tardía.

Dotan et al. ${ }^{26}$ han encontrado que en los pacientes sometidos a bloqueo androgénico precoz, el intervalo entre el desarrollo de metástasis y muerte fue significativamente más corto; no encontrando diferencia en la supervivencia desde el tiempo de la HT en los pacientes que fueron tratados antes o después de la aparición de metástasis. El análisis de las posibles ventajas del tratamiento hormonal precoz frente al tardío debería ser evaluado mediante estudios randomizados prospectivos. La introducción precoz de hormonoterapia puede tener un impacto en la biología tumoral y facilitar la aparición de clones celulares resistentes.

El ensayo de la EORTC 30943 (cerrado en abril 98) pretende investigar el papel de la hormonoterapia introducida de forma inmediata o tardía en pacientes asintomáticos con fallo bioquímico tras prostatectomía y/o radioterapia. 


\section{CONCLUSIONES}

La RT adyuvante es eficaz en pacientes de alto riesgo y con probable presencia de enfermedad subclínica incrementando la supervivencia biológica o clínica con respecto a la cirugía exclusiva. No hay evidencia que incremente la supervivencia.

La RT adyuvante ofrece mejores resultados que la $\mathrm{RT}$ de rescate con una reducción de la tasa de fallos y con un incremento de la supervivencia libre de recaída bioquímica de hasta el 90\% frente al $50 \%$ o menor en la RT de rescate.

La supresión androgénica asociada a la RT adyuvante probablemente puede eliminar la enfermedad sistémica oculta y a la vez conseguir un efecto aditivo en la mejoría del control local y posiblemente incrementar la supervivencia global.

La introducción precoz de la RT de rescate (PSA pre-RT $<2$ ), consigue mejores resultados de supervivencia libre de recaída bioquímica. Peores resultados se obtienen en el grupo de pacientes con persistencia de PSA tras prostatectomía.

Es necesario seleccionar grupos de pacientes, según los factores pronósticos, para determinar si el riesgo predominante es hacia fallo local o a distancia y para predicción de la progresión de PSA tras rescate con RT. La probabilidad de estar libre de progresión se va incrementando a medida que se van asociando los factores de buen pronóstico.

La RT de rescate ofrece la posibilidad de curar pacientes con enfermedad recurrente tras la prostatectomía, incluso en pacientes de alto riesgo de enfermedad diseminada: Gleason 8-10, PSA pre-RT $<2$, con márgenes positivos y asociado a TDPSA > 10 meses, pueden tener una alta probabilidad de estar libre de progresión a los 4 años. Si se asocian estos factores con TDPSA < 10 meses la PLP se reduce de forma considerable.

Es adecuado asociar Hormonoterapia a la RT de rescate o incluir en ensayos clínicos a los pacientes con PSA pre-RT $>2 \mathrm{y} / \mathrm{o}$ con factores pronósticos negativos: Gleason alto con márgenes negativos, afectación de vesículas seminales, TDPSA < 10 meses con márgenes negativos, TDPSA < 10 meses con Gleason 8-10, dada la baja probabilidad de estar libre de progresión con RT exclusiva.
Quedamos pendiente de los resultados de los ensayos actuales (ej: EORTC/RTOG) que ratifiquen o mejoren las conclusiones previamente descritas.

Necesidad de proponer nuevos ensayos randomizados prospectivos para:

a) dilucidar la aportación de la Radioterapia en la prevención de metástasis e incremento de la supervivencia.

b) determinar el efecto aditivo y mejora de resultados al asociar hormonoterapia a la $\mathrm{RT}$ adyuvante o de rescate.

c) establecer el momento óptimo de introducción.

d) resolver si los resultados de la RT de rescate en PSA detectable son comparables a los de RT adyuvante en pacientes de alto riesgo.

Debemos continuar con el análisis, estudio y validación de los factores de riesgo para predicción de fallos locales y de progresión sistémica:

- cambios de PSA, velocidad anual de PSA, pendiente de PSA,

- definición óptima para el cálculo del TDPSA para su aplicación de forma estándar en futuros ensayos clínicos.

Necesidad de avanzar en la incorporación de nuevas dianas terapeúticas e identificación de tratamientos que mejoren la supervivencia en pacientes con factores de mal pronóstico.

\section{REFERENCIAS}

1. Pound CR, Partin AW, Epstein JI, et al. Prostate-specific antigen after anatomic radical retropubic prostatectomy. Urol Clin North Am 1997;24:395-406.

2. Blute ML, Bergstralh EJ, Partin AW, et al. Validation of Partin tables for predicting pathological stage of clinically localized prostate cancer. J Urol. 2000;164:1591-1595.

3. Pound CR, Partin AW, Eisenberger MA, et al.: Natural history of progression after PSA elevation following radical prostatectomy. JAMA 1999;281(17):1591-1597.

4. Anscher MS, Robertson CN, Prostniz R. Adyuvant radiotherapy for pathologic stage T3/T4 adenocarcinoma of the prostate: ten year update. Int $\mathrm{J}$ Radiat Oncol Biol Phys 1995;33:37-43.

5. N. Taylor, JF Kelly, DA Kuban, RJ Babaian, et al. Adyuvant and salvage radiotherapy after radical prostatectomy for prostarte cancer. Int. J. Radiation Oncology Biol Phys 2003;56(3):755-763.

6. Eisenberger MA, Partin AW, Pound C, Roostelaar CV, Epstein J, Walsh P. Natural history of progression of patients with biochemical (PSA) relapse following radical prostatectomy: update. Proc Am Soc Clin Oncol 2003;22:380. Abstract 1527. 
7. Katz MS, Zelefsky MJ, Venkatraman ES, Fuks Z, Hummer A, Leibel SA.Predictors of biochemical outcome with salvage conformal radiotherapy after radical prostatectomy for prostate cancer.Clin Oncol 2003Feb1;21(3):483-489.

8. Andrew J. Stephenson; Shahrokh F. Shariat; Michael J. Zelefsky; Michael W. Kattan; E. Brian Butler; Bin S. Teh; Eric A. Klein; Patrick A. Kupelian; Claus G. Roehrborn; David A. Pistenmaa; Heather D. Pacholke; Stanley L. Liauw; Matthew S. Katz; Steven A. Leibel; Peter T. Scardino; Kevin M. Slawin Salvage Radiotherapy for Recurrent Prostate Cancer After Radical Prostatectomy. JAMA. 2004; 291:1325-1332.

9. Kupelian PA, Katcher J, Levin HS, et al. Stage T1-2 prostate cancer: A multivariate analysis of factors affecting biochemical and clinical failures after radical prostatectomy. Int J Radiat Oncol Biol Phys 1997;37:1043-1052.

10. Epstein JI, Partin AW, Sauvageot J, et al. Prediction of progression following radical prostatectomy: A multivariate analysis of 721 men with long-term follow-up. Am J Surg Pathol 1996;20:286-292.

11. Paulson DF. Impact of radical prostatectomy in the management of clinically localized disease. J Urol 1994;152: 1826-1830

12. Centeno B, Zietman A, Shipley S, et al. Flow cytometric analysis of DNA ploidy, percent S-phase fraction, and total proliferative fraction as prognostic indicators of local control and survival following radiation therapy for prostate carcinoma. Int J Radiat Oncol Biol Phys 1994;30:309-315.

13. Anthony V. D’Amico, MD, Ph.D, Ming-Hui Chen, Ph.D, Kimberly A. Roehl, MPH, William J Catalona, M.D. Preoperative PSA Velocity and the Risk of Death from Prostate Cancer after Radical Prostatectomy .New England Journal 2004;351:125-135.

14. Valicenti RK, Gomella LG, Ismail M, et al. The efficacy of early adjuvant radiation therapy for pT3NO prostate cancer: A matched-pair analysis. Int J Radiat Oncol Biol Phys 1999;45:53-58.

15. Leibovich BC, Engen DE, Patterson DE, et al. Benefit of adjuvant radiation therapy for localized prostate cancer with a positive surgical margin. J Urol 2000;163:11781182 .

16. Anscher MS. Adjuvant radiotherapy following radical prostatectomy is more effective and less toxic than salvage radiotherapy for a rising prostate specific antigen. Int $\mathrm{J}$ Cancer 2001;96:91-93.

17. Catton C, Gospodarowicz M, Warde P, et al. Adjuvant and salvage radiation therapy after radical prostatectomy for adenocarcinoma of the prostate. Radiother Oncol 2001;59: 51-60.
18. Vicini FA, Ziaja EL, Kestin LL, et al. Treatment outcome with adjuvant and salvage irradiation after radical prostatectomy for prostate cancer. Urology 1999;54:111-121.

19. Valicenti RK, Gomella LG, Ismail M, et al. Pathologic seminal vesicle invasion after radical prostatectomy for patients with prostate carcinoma: Effect of early adjuvant radiation therapy on biochemical control. Cancer 1998;82:19091914.

20. Bolla M, Van Poppel H, Van Cangh P, et al. Does postoperative radiotherapy after radical prostatectomy improve progression-free survival in pT3 prostate cancer? EORTC 22911. Abstract. J Clin Oncol 2004;23(Suppl):382.

21. Nudell DM, Grossfeld GD, Weinberg VK, et al. Radiotherapy after radical prostatectomy: Treatment outcomes and failure patterns. Urology 1999;54:1049-1057.

22. Roberts SG, Blute ML, Bergstralh EJ, Slezak JM, Zincke H. PSA doubling time as a predictor of clinical progression after biochemical failure following radical prostatectomy for prostate cancer. Mayo Clin Proc. 2001;76:571-572.

23. Shipley WU, Radiation Therapy Oncology Group: Phase III Randomized Study of Radiotherapy With Or Without Bicalutamide in Patients With PSA Elevation Following Radical Prostatectomy for Carcinoma of the Prostate, RTOG-9601, Clinical trial.

24. King CR, Presti JC, Gill H, Brooks J, Hancock SL. Radiotherapy after radical prostatectomy: does transient androgen suppression improve outcomes? Int J Radiat Oncol Biol Phys 2004;59:341-347.

25. Reynolds W, Wu J, Sun L, et al. Comparison of early versus late hormonal therapy (HT) following early PSA-only recurrence (ePSAR) within the Department of Defense Center for Prostate Disease Research database. Proc Am Soc Clin Oncol 2003;22:392. Abstract 1574.

26. Dotan ZA, Cho D, Rhee AC, et al. The role of hormonal treatment for biochemical recurrence in patients following radical prostatectomy. Proc Am Soc Clin Oncol 2003;22: 381. Abstract 1532 .

Dr J.L. Mengual Cloquell

Servicio de Oncología-Radioterapia

Fundación Instituto Valenciano de Oncología (FIVO) Profesor Beltrán Báguena, 8.

46009 .Valencia

e-mail: jmengual@fivo.org

(Trabajo recibido el 31 enero de 2005) 